\title{
Ueber die Verbindungen des Schwefeleisens mit Stickstoffoxyd;
}

\author{
von Dr. Porzczinsliy aus Odessa.
}

Die Thatsache, dafs Schwefeleisen mit Stickstofoxyd in gewisse lösliche Verbindungen eingeht, ist schon lange bekannt und in den älteren Handbüchern der Chemie bei der Besprechung der Einwirkung des Schwefelwasserstoffs auf die Lösungen der Nitroprussidsalze bereits erwähnt. Doch gebührt das erste Verdienst Roussin, diesen Gegenstund näher ins Auge gefafst zu haben, so wie die Entdeckung, dafs in den betreffenden Verbindungen die Molecule des Eisens vermittelst der Reagentien direct nicht nachzuweisen sind; die Resultate seiner interessanten Beobachtung wurden durch Bussy der französischen Academie vorgelegt und in Compt. rend. XLVI, 224 im Jahre 1858 beschrieben und der Oeffentlichkeit übergeben. Nach dieser Zeit wurde jene Beobachtung nicht wieder erwähnt und gerieth in Vergessenheit, so dafs weder von Roussin noch von anderen Chemikern neue Versuche veröfentlicht wurden *). Auf Veranlassung meines hochverdienten Lehrers, Hrn. Prof. Lehmann in Jena, nahm ich jene Arbeit wieder auf, um ein näheres Studium dieser Verbindungen vorzunehmen, in der Absicht, durch diese neuen Arbeiten einiges Licht über die innere Zusammensetzung der noch lange nicht hinlänglich bekannten Nitroprussidsalze zu erhalten. Indessen hat sich, trotz der Uebereinstimmung meiner Präparate in Bezug aut physikalische Eigenschaften derselben mit jenen von Rou ssin

*) Vgl. Roussin's Ahhandlung in Ann. chim. phys. [3] LII, 285 und diesen Amialen CVII, 120.

D. $\boldsymbol{R}$. 
dargestellten, die Zusammensetzung als eine wesentlich andere erwiesen, so dafs die Formel, die Roussin diesen Verbindungen zuschreibt, als fehlerhaft zu bezeichnen ist.

Roussin stellte seine erste Verbindung dar, indem er die Lösung von salpetrigsaurem Alkali mit Schwefelammonium zusammenbrachte und tropfenweise eine Lösung von Eisenoxyd- oder Eisenoxydulsalz hinzufügte. Nach kurzem Kochen und Durchfiltriren jener Flüssigkeit setzten sich beim Erkalten des Filtrates kleine prismatische Nadeln ab, denen er, ohne näher die procentische Zusammensetzung jener Verbindung anzugeben, folgende Formel zuschreibt :

$$
\mathrm{FeSNO}_{2}+\mathrm{Fe}_{8} \mathrm{~S}_{3} \mathrm{NO}_{2}+\mathrm{Hs} \text {. }
$$

Zur Darstellung der betreffenden Verbindung habe ich nicht allein die oben erwälnnte Methode benutzt, sondern auch, um die umstāndliche Bereitung des dazu nothwendigen salpetrigsauren Alkali's zu umgehen, einen vortheilhafteren Weg einzuschlagen versucht. Als einfachste Bereitung dieser Verbindung kann ich folgende Methode empfelilen : Eisenoxydulsalzlösung wird so lange mit Stickstoffoxyd gesättigt, als dasselbe absorbirt wird, hierauf mit einer Lösung von Schwefelwasserstoff-Schwefelnatrium behandelt, bis eine vollkommen neutrale Reaction resultirt. Nach dem Erwärmen jener Mischung bis auf $100^{\circ} \mathrm{C}$. wird dieselbe vorsichtig durchfiltritt. Man erhält nach diesen Operationen eine schwarzbraune Flüssigkeit, welche beim langsamen Abdampfen schöne Krystallnadeln, die büschelförmig gruppirt sind, alssetzt. Diese Krystalle sind sehr löslich in Wasser und Alkohol und zerniefslich in Aether. - Um dieselben von fremden Salzen zu befreien, löst man diese Krystalle in absolutem Alkohol und überläfst dieselben zu wiederholten Malen der Krystallisation. Nach einiger Zeit setzen sich ziemlich grofse Krystalle von schwarzer Farbe und lelihaftem Pechglanze ab. Dieselben sind dem monoklinoëdrischen System angehörend und zwar von 
tafelförmiger Form. Die Zeichnung Fig. 4 auf Tafel II wird diese Verhältnisse näher erläutern. Es sind dieses Krystalle, bei welchen die Endfläche $o$ gegen die übrigen Flächen entschieden prädominirt; die hier nitcombinirten, mit $s$ bezeichneten Prisnaflächen bilden zu einander die rhombischen Winkel von $124^{\circ}$ und $46^{\prime \prime}\left(56^{\prime \prime}\right.$ ? D. R.). Ihuen untergeordnet sind theils die Flächen einer halben Pyramide $m$, theils die horizontalen Prisinaflächen $r$.

Diese Krystalle zersetzen sich an der Luft höchsı langsauı, wenn nämlich dieselbeı vollkommen rein sind; die ursprünglichen Krystallnadeln jedoch unterliegen einer bei weitem schmelleren Zersetzung, und man bemerkt schon nach einigen 24 Stunden dic Entwickelung des Stickstoffoxyds und den rostfarbigen Anflug derselben. Beim Erwärmen dieser Krystalle bis auf $115^{\prime \prime} \mathrm{C}$. erleiden sie keine Gewichtsveränderung; erwärmt man dieselben jedoch bis $160^{\circ} \mathrm{C}$., so verglimmen sie, wobei Stickstoffoxyd und $W$ asser entweichen. Die quantitative Analyse dieser bei $100^{\prime \prime} \mathrm{C}$. getrockneten Krystalle ergab folgende procentische Zusammensetzung :

$\begin{array}{lcc} & \text { Gefunden } & \text { Berechnet } \\ \text { Eisen } & 39,90 & 40,00 \\ \text { Schwefel } & 21,04 & 22,87 \\ \text { Stickstoffoxyd } & 28,69 & 28,56 \\ \text { Wasser } & 8,22 & 8,57\end{array}$

Aus dieser Zusammensetzung läfst sich folgende Formel zusammenstellen :

$$
\mathrm{FeSFe}_{2}\left(\mathrm{NO}_{2}\right)_{2}+2 \mathrm{HO} \text {. }
$$

Diese Formel entspricht auch den nachfolgenden Verhindungen.

Diese Verbindung wird im Allgemeinen durch stärkere Säuren zersetzt. Es entwickelt sich dabei Schwefelwasserstoff, ohne jedoch die Lösung zu entfärben. Das schwarze Colorit derselben verschwindet hingegen beim Erwärmen. 
Diese braune Färbung rührt offenbar von dem in neugebildetem Eisenoxydulsalz gelöstem Stickstoffoxyd her.

Neutrale Alkalisalze, so wie die Salze der alkalischen Erden sind ohne kinwirkung, die der schweren Metalle hingegen wirken zersetzend; besonders gilt dieses von den $\mathrm{Me}-$ tallen, die in saurer Lösung durch Schwefelwasserstoff gefällt werden. Man bemerkt sogleich nach dem Zusammenbringen einer Salzlösung der hetreffenden Metallgruppe mit nitroschwefeleisensaurer Schwefeleisenlösung die Entwickelung des Stickstofloxyds und einen aus der Schwefelverbindung des angewandten Metallsalzes bestehenden Niederschlag, indem das Eisen durch die freigewordene Säure in löslichen Zustand übergeführt wird. Aummoniak und Schwefelammonium zersetzen diese Verbindung mur beim Kochen und dann äufserst schwach. Aetzende Alkalien, so wie ihre Schwefelverbindungen greifen dieselben an und bilden mit diesen unter partieller Zersetzung derselben eigenthümliche Verbindungen, die sogleich näher beschrieben werden sollen.

Setzt man zu der kochenden Lösung des nitroschwefeleisensauren Schwefeleisens allmälig Aetzkali, so trübt sich Anfangs die Lösung und es scheidet sich bei fortgesetztem Kochen ein rother Niederschlag, aus Eisenoxyd bestehend, aus. Dabei findet keine Ammoniakentwickelung statt, wie Roussin in seiner Abhandlung (siehe oben) angiebt; wohl aber geschicht es, wenn Aetzkali in grofser Menge bei stürmischem Kochen zugesetzt wird. Aus dem Filtrat setzen sich, nachdem abgedampft und die zurückbleibende concentrirte Flüssigkeit unter der Luftpumpe üher Schwefelsäıre stehen gelassen wird, schöne schwarze Krystalle ab, welche klinorhomboidisch mit klinorhombischem Habitus sind.

Diese Krystalle sind der Luft ausgeselzt äufserst zersetzbar, indem sich dieselben sogleich mit einer Schicht von Eisenoxyd bedecken. Beim Erwärmen bis auf $100^{\circ} \mathrm{C}$. verAnn. d. Chemie u. Plinrm. ExxV. Hd. 3. Heft. 
lieren dieselben 16,5 pC. Wasser, bei stärkerem Erwärmen erfolgt eine ziemlich heftige Explosion. Sie sind fast in gleichen Theilen Wasser löslich, schwerer dagegen in Alkohol und vollkommen unlöslich in Aether.

Durch wiederholtes Umkrystallisiren aus Wasser erhaltene und bei $100^{\prime \prime} \mathrm{C}$. getrocknete Krystalle wurden der chemischen Analyse unterworfen, wobei sich ilire procentische Zusammensetzung wie nachstehend erwies :

$\begin{array}{lcc} & \text { Gefunden } & \text { Bercchnet } \\ \text { Eisen } & 28,417 & 27,58 \\ \text { Schwefel } & 21,405 & 23,64 \\ \text { Kalium } & 18,385 & 19,21 \\ \text { Stickstoffoxyd } & 30,080 & 29,55 .\end{array}$

Dieser Zusammensetzung entspricht folgende Formel : $\mathrm{KS}, \mathrm{Fe}_{2} \mathrm{~S}_{8}\left(\mathrm{NO}_{2}\right)_{2}$.

Roussin schreibt der entsprechenden von ihm dargestellten Natriumverbindung folgende Formel zu :

$$
\mathrm{Fe}_{2} \mathrm{~S}_{3} \mathrm{NO}_{4}, 3 \mathrm{NaS} \text {. }
$$

Dic: Lösung des nitroschwefeleisensauren Schwefelkaliums wird durch die Einwirkung der ätzenden Alkalien nicht verändert. Die Säuren dagegen zersetzen dieselben sehr energisch, doch hängen die Zersetzungsproducte sowohl von der Concentration der Säure, als auch von der Qualität derselben ab. Concentrirte Säuren wirken im Ueberschufs zugesetzt vollkommen zersetzend; werden ganz verdüınte Säuren allmälig zugesetzt, so bemerkt man eine sehr schwache Schwefelwasserstoffentwickelung, die Mischung verändert die Farbe und geht in ein dunkelbraunes Colorit über; wird diese Flüssigkeit abgedampft, so setzen sich beim Erkalten Krystalle von Nitroschwefeleisenschwefeleisen nebst einem unbedeutenden liükkstand von Eisenoxyd ab. Eben so verhält sich die Kohlensäure. Werden die Säuren auf einmal bis zur Neutralisation zugesetzt und die Flüssigkeit gleichzeilig erwärmt, so ent- 
wickelt sich Schwefelwasserstoff in grofser Menge und es fällt ein kaffeebrauner Niederschlag nieder, der bei fortgesetztem Erwärmen schwarz wird. Dieser Niederschlag ist Anfangs in Wasser unlöslich, zersetzt sich jedoch nach einiger Zeit, indem ein Theil desselben in Wasser wieder aulgelöst wird. Auch Alkohol und Aether nehmen diesen Körper theilweise in Lösung. Wird der kaffeebraune Niederschlag sogleich, nachdem derselbe vermittelst der Säure gefältt wurde, zu wiederholten Malen mit Schwefelalkalien versetzt, so löst sich derselbe vollkommen auf und bildet das ursprüngliche Salz.

Gegenwärtig bin ich mit dem weiteren Studium dieser interessanten Verbindungen beschäftigt, und hoffe in der Kürze ausführlicher über diesen Gegenstand berichten zu können.

\section{Von dem Uebergang aus der Acrylreihe in die Reihe der Fettkörper, und ungekehrt;}

\section{von Ed. Linnemann.}

1) Verhalten des Acetons gegen Brom.

Bei Gelegenheit einer Mittheilung über das Verhalten des Benzophenons gegen Natriumamalgam habe ich vor Kurzem auch einige Betrachtungen über die Entstehung des Propylalkohols aus Aceton angestellt und die Ansicht ausgesprochen, dafs sich das Aceton, wie in anderen Fällen, so auch dem Natriumamalgam gegenüber wie sein Isomeres, der Allylalkohol, verhalte. 